\title{
Envejecimiento del personal
}

Sergio Castrillon ${ }^{1}$

Recibido: Enero 2006

Docente Escuela de Administración, Universidad Eafit, Colombia. Magíster en Estudios Políticos; $\mathrm{PhD}$ ( c ) en Administración, HEC - Montréal. El presente documento constituye un capítulo de su Tesis Doctoral.

Aceptado: Marzo 2006 
El envejecimiento de la población es un fenómeno humano que se expresa a nivel colectivo e individual, y que impacta significativamente los escenarios y las dinámicas laborales. Las personas, organizaciones, naciones y comunidad internacional, deben enfrentar interrogantes inéditos que emergen a medida que la población humana se multiplica y envejece.

En términos generales podríamos hablar de cambios estructurales a nivel social, frente a los cuales las ciencias administrativas deben (así como los departamentos de recursos humanos RRHH- dentro de las empresas) permanecer particularmente atentos, pues ciertas manifestaciones sociales del envejecimiento repercuten directamente en las organizaciones económicas. $\mathrm{Al}$ examinar detalladamente la literatura existente, hemos encontrado que el envejecimiento se ha problematizado desde diversas perspectivas, tales como: las mutaciones de la fuerza laboral; los costos y beneficios del envejecimiento de los trabajadores; los desafíos de la jubilación; la escasez de personal experimentado; así como varias consideraciones organizacionales que discutiremos en las secciones siguientes.

\section{Las mutaciones de la fuerza laboral}

Las tendencias demográficas mundiales de incremento en la longevidad de las personas, disminución de las tasas de natalidad, así como inmigración restringida; conducen al fenómeno del envejecimiento de la 'fuerza la- boral', que a su vez acarrea consecuencias que afectan directamente la gestión del personal de las empresas. los recursos humanos. Un descripción adecuada de su relevancia para el campo de la gestión, la encontramos en Management Review, -publicación representativa de la literatura administrativa, en la cuál se explica como varias problemáticas incrementaban su visibilidad en función del envejecimiento de la fuerza laboral: la "escasez para el reclutamiento, retiro obligatorio, discriminación por la edad", son algunos de los factores que salían a la superficie (Capowski, 1994).

Algunos autores catalogan al cambio demográfico de los últimos años como uno de los "fenómenos más sorprendente en la administración de recursos humanos" (Crampton, Hodge, \& Mishra, 1996), amplificado además por la singularidad en términos de población que representa la generación de los baby boomers.

Efectivamente, los baby-boomers constituyen una mutación elocuente en el devenir demográfico de los países de occidente; nacidos entre 1946 y 1964 , ellas y ellos, encarnan el fruto de los dramáticos cambios (de actitud, culturales, económicos...), que acompañaron la posguerra. Los 'boomers' simbolizan también la urgencia de los desafíos demográficos estructurales que enfrenta la humanidad, especialmente en los países más avanzados económicamente. Con la llegada a sus sesenta años (en el 2006), los retos y oportunidades que reflejan una gran transición laboral se hacen inaplazables. 
Para conservar la perspectiva histórica de las encrucijadas actuales, recordemos que desde la década de los ochenta diversas voces anunciaban los cambios por venir en cuanto a las actitudes y perfiles de los empleados, su interacción con la tecnología, impacto en las expectativas y dinámicas de jubilación, problemas de discriminación, etc. (Menefee, 1983), (Kim Cameron \& Zammuto, 1983), (Hammett, 1984), (Coates, 1987). A medida que la población sigue envejeciendo, muchos de los asuntos permanecen aún irresueltos, lo cual mantiene vigentes los desafíos y oportunidades que enfrentan los departamentos de RRHH de las empresas.

Así pues, a pesar de los grandes cambios geopolíticos (e.g. fin de la guerra fría, emergencia de bloques económicos, discursos antiterroristas), y las revoluciones tecnológicas; los retos demográficos y sus eventuales impactos a nivel organizacional no han disminuido; por el contrario se han acentuado.

Un artículo aparecido en Benefits Quaterly nos ofrece un abanico de advertencias y recomendaciones que resumen acertadamente la permanencia de preocupaciones en ambas épocas. Entre sus advertencias a los empleadores, encontramos la urgencia de atender cambios generados por las mutaciones demográficas y de la fuerza laboral, tales como el aumento de la población de más de 60 años, de la edad promedio de la masa laboral, la escasez de personas jóvenes entrando al mercado laboral; así como el in- cremento de los costos médicos, y eventualmente de los salarios, pues estos deberían aumentarse tanto para atraer los mejores jóvenes, como para mantener los antiguos empleados (Rappaport, 1988).

Las recomendaciones de Benefits Quaterly, aunque insuficientes para resolver las exigencias actuales $-\mathrm{y}$ sobretodo, insuficientes para satisfacer a todos los grupos de interés involucrados- permanecen vigentes, a saber: establecer programas para retardar el retiro o para efectuarlo gradualmente, establecer mecanismos de respaldo para los empleados que ayudan a cuidar a sus padres ancianos -e.g. servicios de referencia, grupos de apoyo, flexibilidad en los horarios, etc. (Rappaport, 1988).

En términos generales podemos afirmar que las advertencias anunciadas, las cuestiones presentes, y los dilemas futuros, abarcan por igual -aunque de manera distinta-al sector público y privado. A pesar de las diferencias en las dinámicas de cada mercado laboral -en niveles de salarios, de flexibilidad (i.e. estabilidad) y sujeción a la legislación laboral (contratación de minorías visibles, políticas anti-discriminación, escenarios de sindicalización); ambos sectores presentan desafíos cualitativamente similares.

Por ejemplo, los elementos de transición que los especialistas recomiendan prever, son comunes a ambos escenarios laborales (sector público y privado): la preocupación respecto a los 'costos salariales' y de 'retiro', respecto a las posibles alteraciones de 
salud y sus impactos financieros; a los niveles de competencia y de desempeño; a la gestión del relevo generacional, la transmisión de saberes, etc.

No obstante la similitud, la existencia de matices y dinámicas propias a cada sector, estimula pensar en la posibilidad de efectuar investigaciones comparativas alrededor de asuntos comunes a los dos sectores; eventualmente entre diversos países. Así, podrían entrelazarse las formas de abordar el terreno, los motivos que inicialmente despiertan el interés (que pueden diferir en los contextos público y privado), y por supuesto, descubrir las distintas lógicas subyacentes.

Con el propósito de resaltar otros elementos que han suscitado atención, a continuación evocamos algunos ejemplos provenientes de distintos sectores; ejemplos que ponen de manifiesto vectores útiles para el análisis comparativo de las mutaciones de la fuerza laboral, a la vez que nos permiten interrogarnos acerca de la presencia de racionalidades diversas.

Respecto al impacto de los cambios demográficos en el sector público, encontramos estudios relevantes; por ejemplo en relación a los programas de entrenamiento y desarrollo, los regímenes de jubilación, y la legislación anti-discriminación (Elliott, 1995). También de interés académico resulta el estudio realizado por Blue Woodridge entorno a las agencias del gobierno estadounidense, examinando la conjugación de los cambios demográficos y los esfuerzos por incrementar el uso tecnológico (como estrategia de productividad); evaluando los factores de éxito potencial, así como las limitaciones. Los hallazgos principales, sugieren que los administradores deben ser sensibles a las diferencias de cada segmento demográfico, pues la forma de servirse de la tecnología puede variar en función de los estilos cognitivos, de la edad, de diferencias de género, étnicas, etc. (Woolridge, 1994).

Dicho sea de paso que la tecnología no constituye ninguna panacea, por el contrario puede ser fuente de rupturas. De todas formas, es una variable de gran interés, pues interviene de múltiples maneras en las posibilidades de acción e intervención de los empleados de todas las edades.

Por ejemplo, el estudio de Judi Brownell (sobre los administradores de hospitales) demuestra como la presencia simultánea de la tecnología y del envejecimiento de la población acarrea nuevos desafíos, pues 'aumenta las demandas de servicio de sus trabajadores del sector servicios' y la necesidad de entender como las personas de avanzada edad 'se adaptan a nuevas relaciones, nuevas conductas y nuevos servicios' (Brownell, 1994).

La introducción de nuevas tecnologías, abre toda una gama de nuevas posibilidades, (que contienen a la vez promesas y riesgos) pero que de ninguna manera sustituyen la necesidad de instaurar mecanismos de comunicación efectiva hacia todas las personas que participan en los procesos organizacionales. Especialmente cuando las personas ganan en años, es cuando más conviene refinar contextos de escucha 
efectiva (y afectiva) de todos los interlocutores, sean aquellas personas que ofrecen el servicio o los que lo consumen; por eso podemos extrapolar la conclusión de Brownell y afirmar que los managers de todos los sectores y edades deben asumir tareas de motivación, coaching y mentoring para prestar mejores servicios.

\section{Preocupaciones alrededor del mundo}

Organizations were less likely to be adjusting training methods to accommodate the needs of older employees. There was little attempt to provide age awareness training to managers of older employees. The challenge for organizations will be to close the gaps that currently exist between the practices that are important in retaining older managerial and professional employees and the extent to which organizations are engaging in these practices. Ensuring access to training, customizing training methods, and providing age awareness training require immediate attention.

Marjorie Amstrong-Stassen, Andrew Templer (2005)

La actividad académica en el campo de RRHH esta estrechamente relacionada con las urgencias y acciones de las empresas. Muchas de las preocupaciones son similares entre las empresas y países de occidente; que además de competir en los mismos escenarios económicos, enfrentan retos análogos en materia de población. Los siguientes párrafos proponen poner de manifiesto algunos de los factores que jalonan la investigación académica y las acciones de las empresas en el contexto de la mundialización contemporánea.

Empecemos por Canadá; donde en un estudio paradigmático Marjorie Amstrong-Stassen y Andrew Templer (2005) evalúan las gestiones que los ejecutivos de recursos humanos respecto a los retos del envejecimiento de la población. Su investigación demuestra que menos de $10 \%$ de las empresas están comprometidas significativamente en la implementación de programas de entrenamiento, y que un porcentaje inferior, ajusta sus métodos a las necesidades de los empleados de mayor edad. Los autores señalan el desafío de 'cerrar las brechas' entre las prácticas existentes y el nivel de compromiso necesario para retener los profesionales y administradores más antiguos. Entre las sugerencias concretas encontramos la necesidad de mejorar los programas de desarrollo de personal, ofreciendo talleres de sensibilización respecto a la edad, ajustando los programas de entrenamiento a las necesidad de los mayores y facilitando su acceso (Armstrong-Stassen \& Templer, 2005).

Aunque muchos autores denuncian la escasez de información y el hecho de que las investigaciones no hacen justicia a la importancia del segmento 'mayor' de las poblaciones; es posible encontrar ejemplos de varios países que nos muestran la persistencia de los desafíos académicos y empresariales así como algunas de las estrategias implementadas por las organizaciones. 
Por ejemplo, Margaret Patrickson y Rob Ranzijn nos muestran (desde Australia), la ausencia de mecanismos que permitan intercambiar las posturas e integrar las expectativas de los distintos actores involucrados en el proceso, así como la persistencia de las actitudes negativas hacia la contratación de las personas mayores. Para mantener su rentabilidad las empresas han implementado tácticas de retiro por fases, acciones de mejoramiento en salud ocupacional y de seguridad dirigidas a retener a los empleados que la organización desea mantener. Respecto a los empleados cuyas habilidades no son estratégicas para la organización, se observa la implementación de provisiones de redundancia (Patrickson \& Ranzijn, 2004), es decir su reemplazo directo sin consideraciones particulares por los individuos.

Respecto a los EEUU encontramos que a pesar de los anunciados cambios demográficos, los esfuerzos de reclutamiento y retención en el sector de salud han sido insuficientes, en cantidad y en el ajuste del diseño a las particularidades de cada franja de trabajadores (Barney, 2002). Para la economía en general encontramos que según lo observan algunos autores, las recomendaciones de la Oficina de Manejo de Personal, han oscilado entre los esfuerzos de motivación, integración laboral de las mujeres y minorías, así como del reclutamiento y retención (Kristi Cameron, Jorgenson, \& Kawecki, 1993).

En Japón las predicciones de escasez de mano de obra han provocado cambios interesantes desde hace más de una década. Por ejemplo, un académico japonés reportaba en el Journal of Small Business Management los cambios de una empresa centenaria, donde los 'seniors' eran asignados a cargos con responsabilidades de especialistas en vez de recibir puestos con títulos simbólicos y poca acción (Lee, 1991).

En general vemos que los cambios demográficos se manifiestan con vehemencia en las empresas de distintos países, y no sólo de manera general sino acarreando preocupaciones específicas, respecto a las cuales el área de RRHH ha sido particularmente receptiva. A continuación discutimos algunas de ellas.

\section{Costos y beneficios de la fuerza laboral envejeciente}

Sin lugar a dudas uno de los elementos que suscita mayor interés es el relacionado con el impacto económico del envejecimiento. Cálculos, pronósticos y especulaciones entorno a los costos de la 'mano de obra' según su edad, así como respecto a la carga prestacional (de seguridad social) de los jubilados, constituyen el tema central de múltiples estudios.

Las discusiones oscilan entre dos extremos diametralmente opuestos: de un lado las imágenes positivas que resaltan las contribuciones potenciales de los viejos en términos de rentabilidad y ahorro de nuevos gastos de contratación; y de otro lado los estereotipos negativos de baja productividad, fragi- 
lidad médica y la consecuente carga financiera. Un buen ejemplo de esta dualidad es el artículo de Public Personnel Management donde sobresalen las temáticas de salud y los impactos financieros del cuidado de los viejos, al tiempo que evocan el talento 'disponible' pues son confiables, bien entrenados y bien educados para el trabajo(Crampton et al., 1996).

Tal dualidad, se explica por la tensión entre las pretensiones simultáneas por aumentar los niveles de desempeño y por disminuir los costos de contratación. Las creencias populares suelen sugerir que los viejos producen menos y que resultan más costosos a las organizaciones. En este sentido el desafío para las ciencias administrativas puede postularse en dos sentidos: 1) identificar un acervo confiable de evidencias que facilite la toma de decisiones; 2) cuestionar las lógicas inherentes a las argumentaciones que se presentan, para balancear sus efectos en el corto y en el largo plazo.

Concretamente, resulta imprescindible anticipar los efectos substanciales de las medidas corto-placistas que instrumentalmente buscan ahorrar los 'costos' de nomina, quizás erosionando la capacidad de la empresa de generar valor en el largo plazo. También resulta ineluctable interrogar críticamente los fundamentos axiológicos y epistémicos de los sistemas contables que condicionan nuestra percepción de los viejos, viéndolos como un costo y ni siquiera como un 'activo/recur- so' organizacional.

El Internacional Journal of Manpower ilustra las contradicciones que pueden encontrarse al abordar los costos de nómina de los empleados más antiguos. A partir de su análisis de costos y beneficios económicos de reclutamiento, entrenamiento, ausentismo y accidentes laborales, Lobby Brooke - la autora del artículo- descubre que el balance es cuantitativamente favorable para los viejos: en términos monetarios reportan más ingresos que egresos, en cifras absolutas y también comparativamente con el segmento de los jóvenes. Además aportan otro tipo de beneficios no necesariamente cuantificables: optimización del conocimiento, conservación de habilidades, perspectivas amplias en la toma de decisiones, prevención de accidentes laborales (Brooke, 2003).

A partir de los antecedentes investigativos encontrados, nos parece que existen vacíos importantes respecto al tema de los costos y aportes de los empleados de mayor edad. Además de la ausencia de conclusiones definitivas, también creemos que a menudo se esquivan preguntas de mayor profundidad y responsabilidad social. Por ejemplo, ¿como evitar el determinismo de las urgencias corto-placistas, procurando por el contrario asegurar la viabilidad de las empresas en el largo plazo y el respeto por todos los empleados más allá de 'significación monetaria'? Las oportunidades de investigación son múltiples. 


\section{El retiro laboral}

By now it is likely that you have heard the statistics, the predictions and the dire warnings. The aging of the largest generation the United States has ever known will affect every social institution from employment to health care. [...]

(Kiyonaga, 2004)

Las preocupaciones acerca de la jubilación constituyen otro vector de interés significativo. Nancy Kiyonaga nos recuerda que "hoy es el mañana del cual nos preocupábamos ayer”, y que aun persisten incertidumbres acerca de cuáles serán los impactos específicos del retiro laboral de los baby boomers, la generación más grande que los EEUU hayan conocido (Kiyonaga, 2004) y que provoca repercusiones en el resto del mundo. La presencia de incertidumbre engendra la necesidad de incorporar flexibilidad en el diseño e implementación de los sistemas de jubilación.

Las exigencias de flexibilidad se extienden a todos los niveles: sociales, organizacionales e individuales; lo cual reclama la intervención de distintos tipos de conocimientos, a saber: socio-político-económicos, administrativos y sicológicos. Este tipo de desafíos debe extenderse tanto a la academia, como a las gerencias de 'recursos humanos'. Así, el llamado de Benson Rosen y Thomas Jerdee por incrementar los esfuerzos investigativos y educativos sigue vigente (1989), pues cada ola generacional trae sus propias particularidades, que además es preciso ajustar según los sectores económicos y países.
Por ejemplo, la revista Affaires Universitaires reporta algunas de las controversias y dificultades enfrentadas en el proceso de negociación mediante el cual la Universidad de Toronto logró el primer acuerdo (entre todas las universidades canadienses) para eliminar la cláusula de retiro obligatorio que afectaba a sus docentes $(\mathrm{Mu}-$ 1lens, 2005). El hecho de que este acuerdo sólo se pudiera concretar 11 años después que en los EEUU, testimonia de la dificultad para ofrecer alternativas flexibles (tales como la jubilación temprana, tardía, por fases o parcial) frente al retiro regular; incluso en los sectores más ilustrados, y que se ven más directamente afectados por la fuga de cerebros, como consecuencia de la rigidez laboral en materia de jubilaciones.

Los niveles de flexibilidad deseables, así como la brecha entre ellos y los actualmente disponibles, sigue siendo uno de los motivos de interés práctico y teórico para las ciencias administrativas. De igual manera que las acciones y reacciones de los distintos agentes involucrados.

Las discusiones no se limitan a Norteamérica, por ejemplo Gordon Rabey, desde Nueva Zelanda, adopta una perspectiva internacional y señala la importancia de introducir cláusulas de flexibilidad respecto a la jubilación en los contratos de trabajo. Su argumentación es interesante porque desborda la dimensión económica y rescata las contribuciones positivas que pueden esperarse de las personas de edad; exhibiendo los beneficios mu- 
tuos de permitirles conservarse laboralmente activos (Rabey, 2005).

\section{Escasez de personal calificado}

Entre los principales factores de preocupación de las organizaciones encontramos la necesidad de enfrentar la insuficiencia de personal calificado. En los próximos años gran cantidad de personas con talento cultivado y valiosa experiencia acumulada se retiraran de sus empleos regulares, provocando enormes dificultades para las empresas que necesitan reemplazarlos. El vacío será especialmente sensible en aquellos oficios que requieren más crystallized intellectual abilities $-G c-$, (habilidades intelectuales cristalizadas; concepto que discutiremos más adelante, en la sección que trata acerca de la motivación).

Las dificultades de encontrar personal calificado que pueda remplazar la gran ola de jubilaciones, constituyen otra de las razones por las cuales las ciencias administrativas se han interesado en los efectos del envejecimiento en diversos países, examinando las variaciones en sectores según el tipo de competencias requeridas y la sensibilidad social que les acompaña. Un ejemplo significativo surge en el área de la salud; donde la escasez de médicos y enfermeras se ha manifestado críticamente en muchos países que no alcanzan a reemplazar a sus profesionales, ni en términos absolutos, ni en términos relativos, frente al incremento continuo de la masa poblacional.
A pesar de las advertencias de décadas anteriores, la situación aún está lejos de solucionarse. El diagnóstico que Richard Hader realiza para el sector salud de los EEUU puede extrapolarse al resto del mundo y a muchas otras profesiones; en ese orden de ideas también debemos acoger su interpelación a "desarrollar estrategias creativas de retención de (empleados) de edad con el objetivo de minimizar los efectos de la escasez de personal" (Hader, 2005).

Al analizar al Japón y sus déficits de personal calificado en diversos sectores industriales, Jenny McCune nos proporciona un ejemplo que, desde otro país y sector, nos confirma la importancia estratégica de asegurar el compromiso y cooperación de los empleados mejor calificados, en una época donde sus expectativas van cambiando y no se satisfacen con la simple estabilidad laboral. Desde este punto de vista -i.e. en el contexto Japonés, replicable a otros escenarios-, el desafío para los administradores de RRHH consiste en 'atraer y mantener los trabajadores que hacen falta a la empresa,' incorporando los avances tecnológicos, y los flujos migratorios (McCune, 1990). Lo interesante en el ejemplo japonés, es que aunque la idea del empleo vitalicio es cada vez más difícil de garantizar, aún se trata de respetar, procurando así mantener los más altos niveles de confianza que a su vez garanticen la lealtad de los demás trabajadores.

Como podemos observar, los factores macro sociales y económicos que 
acompañan el envejecimiento humano e impactan directamente a las organizaciones, son múltiples y de diverso orden; cada uno de los cuales merece a su vez consideraciones especiales. La sección siguiente explora la perspectiva organizacional, para complementar la mirada de los grandes agregados.

\section{Perspectiva organizacional}

Asumir los puntos de vista organizacionales nos permite comprender mejor como se entrecruzan los niveles de preocupación social e individual; y la forma en que los académicos de la administración han abordado las problemáticas relacionadas con el envejecimiento.

Por ejemplo, el avance o estancamiento en la carrera profesional, configura una vivencia personal que desborda la esfera laboral e impacta diversas dinámicas sociales. De entrada, habría que reconstruir la definición misma de dichos eventos, sería preciso examinar sus múltiples interpretaciones; contrastar la noción de 'éxito' con la de 'calidad de vida', etc.

$\mathrm{Al}$ considerar las preocupaciones organizacionales no podemos ignorar las voces expresadas en Harvard Business Review (pues al exhibir las posiciones del main-stream, nos alertan acerca del imperativo crítico-intelectual, de deconstruir posturas dominantes buscando la construcción de realidades más humanas).

Volviendo a HBR, que publica uno de los artículos pioneros al abordar el problema del envejecimiento -desde la mirada de la empresa-, Arch Patton anticipaba las preocupaciones alrededor de los cambios en la 'motivación y productividad' de los mandos medios, así como los posibles incrementos en los costos de pensiones. Resulta curioso que aunque él identificaba factores macro como causantes de la situación (e.g. la inflación, las variaciones demográficas); las soluciones las recargaba todas sobre los niveles micro, sugiriendo que las compañías podían: " 'comprar' el retiro anticipado de algunos de sus ejecutivos, utilizar promociones 'cambiando los 'rotulos', y encontrando otros símbolos de logro 'cargados' de emoción" (Patton, 1981).

Aunque la situación ha mejorado en los últimos 20 años, muchos de los desafíos de entonces continúan aumentando en función de las crecientes presiones demográficas; y de la necesidad de asumir espacios de reflexión crítica; de equilibrar las grandes transformaciones demográficas con las capacidades individuales y las responsabilidades organizacionales. Al observar el funcionamiento actual de las grandes empresas, obsesionadas por la rentabilidad financiera (que ahoga las búsquedas sensatas de creación de valor industrial), nos percatamos de la contundente actualidad de las palabras de Edmund Metz, quién denunciaba el bajo peso estratégico otorgado a la variable de recursos humanos y la necesidad de que las organizaciones asumieran responsabilidades educativas respecto a los empleados de 
todas las edades (Metz, 1984) -a los nuevos para perfilarlos mejor dentro de la empresa y a los antiguos para mantener sus competencias.

Los pronósticos del pasado y las realidades del presente nos cuestionan de distintas maneras: ¿Qué hacen las empresas actualmente para enfrentar el impacto de las variables demográficas?, ¿en que sentido incorporan visiones de largo plazo, para garantizar su sostenibilidad futura?, ¿Cómo se incorporan las dimensiones humanas en las formulaciones estratégicas?, ¿Qué cambios a nivel de políticas y programas organizacionales se han estudiado/formulado?, ¿Qué actividades educativas, por ejemplo para educar a los empleados, y adaptarles a los adelantos tecnológicos y a las exigencias de los nuevos entornos?, ¿Qué hacen las empresas para evitar la discriminación?

En las secciones siguientes examinamos las formas en que el conocimiento administrativo ha abordado las inquietudes anteriores, derivadas todas del envejecimiento del personal.

\section{¿Cómo se está 'administrando' la fuerza laboral?}

Una visión panorámica de cómo despuntaba el manejo de una fuerza laboral en proceso de envejecimiento lo encontramos en el Review of Public Personnel Administration, donde Jonathan West y Evan Berman investi- gan la 'administración estratégica de los trabajadores de más edad' según las acciones implementadas por los administradores de recursos humanos en las poblaciones de más de 50.000 personas en los EEUU -a partir de una muestra representativa de todo el país(West \& Berman, 1996).

Los académicos encontraron que las estrategias gravitan alrededor de cuatro áreas fundamentales: 1) relaciones de respaldo en el lugar de trabajo, 2) entrenamiento, 3) desarrollo de carrera, 4) evaluación del desempeño; aunque los autores aclaran que prácticamente en todas las áreas los esfuerzos manifestados son insuficientes. (West \& Berman, 1996).

Es interesante observar que en cierta medida los desarrollos teóricos también pueden clasificarse alrededor de estos temas, lo cual nos permite adoptar un marco de referencia para la presentación de distintas aproximaciones al fenómeno del envejecimiento humano.

Las páginas que siguen se articulan entonces alrededor de las problemáticas arriba explicitadas; inicialmente presentamos material específico entorno a la evaluación del desempeño; después la literatura entorno a la motivación y el entrenamiento; en tercer lugar algunas dimensiones relacionadas a la perspectiva individual que aportan otra mirada sobre el desarrollo de carrera; finalmente examinamos la discriminación por la edad en tanto patología que afecta las relaciones laborales. 


\section{Competencias y desempeño}

Las competencias y aportes potenciales de los empleados de mayor edad constituyen un tema que despierta gran interés en las ciencias administrativas. Las investigaciones suelen buscar satisfacer las preguntas de la empresa respecto a como 'optimizar' las contribuciones de todos sus empleados, en especial respecto a los viejos, pues como hemos dicho antes; a medida que la población envejece ellos constituyen un componente significativo de la fuerza laboral, son necesarios y a menudo representan un 'costo más alto'. Todos estos factores, además de los desafíos de integración intergeneracional, conducen a preocuparse por los viejos dentro del escenario laboral.

A pesar de los prejuicios, las evi- dencias empíricas demuestran el buen desempeño de los adultos mayores en actividades de interés para las empresas; (Bushko \& Raynor, 1999), (Griffin, 2005), (Kanfer \& Ackerman, 2004), etc. Gran parte de la aprehensión se nutre de los prejuicios, prejuicios que en ocasiones son difíciles de superar, pues en cierta medida se encuentran relacionados con nuestros propios temores a la vulnerabilidad y deterioro que acarrea el proceso biológico que acompaña al paso de los años.

Varios de los artículos que encontramos referencian el trabajo de Peter Warr, quien propone una conceptualización de cuatro categorías potenciales que relacionan la edad con el desempeño laboral (Kanfer \& Ackerman, 2004), (Patrickson \& Hartmann, 1995). La siguiente tabla resume las ideas de Warr,-según pudimos consultarlas en las fuentes secundarias mencionadas-:

\begin{tabular}{|l|l|l|l||l|}
\hline & Tipo A & Tipo B & Tipo C & Tipo D \\
\hline \hline Tipo de Actividad & $\begin{array}{l}\text { Comprensión } \\
\text { Verbal }\end{array}$ & $\begin{array}{l}\text { Memoria } \\
\text { Inmediata }\end{array}$ & $\begin{array}{l}\text { Razonamiento } \\
\text { Complejo }\end{array}$ & $\begin{array}{l}\text { Velocidad de Reacción, } \\
\text { Memoria de Trabajo, }\end{array}$ \\
\hline $\begin{array}{l}\text { Rotulo/denominación } \\
\text { científica }\end{array}$ & $\begin{array}{l}\text { Inteligencias } \\
\text { Cristalizada } \\
\text { Relación del } \\
\text { desempeño con la edad }\end{array}$ & --- & --- & Inteligencia Fluida \\
Mejora & $\begin{array}{l}\text { No hay } \\
\text { relación }\end{array}$ & $\begin{array}{l}\text { No hay } \\
\text { relación }\end{array}$ & Relación negativa \\
\hline
\end{tabular}

En general las evidencias empíricas nos muestran que, contrario a ciertos prejuicios negativos, los viejos no tienen obstáculos inherentes en aprender y aumentar el desempeño laboral. Incluso en las actividades de tipo 'inteligencia fluida', un entrenamiento bien dirigido permite que con el paso de los años también se mejore la me- moria de trabajo y la velocidad de reacción. Además de algunos estudios que evalúan positivamente los resultados de los viejos, también se presentan argumentos indicando la capacidad ociosa del cerebro que es posible actualizar; es decir que usualmente habría cierta potencialidad a utilizar para evitar el deterioro neuronal. 
Para la empresa el balance es positivo, de hecho, según lo indican Margaret Patrickson y Linley Hartman (1995), la capacidad de procesar y analizar información permanece estable, al menos durante toda la vida laboral, y cualquier deficiencia en velocidad o precisión (remediable mediante practica y entrenamiento) es compensada por mayor estabilidad y autonomía en el trabajo.

Para resolver las discrepancias respecto al desempeño laboral de los adultos mayores aun falta mucho terreno por recorrer. Avanzar en ese sentido implica diferenciar el tipo de actividades que se pretende evaluar y por supuesto, conciliar distintos horizontes de tiempo. En ocasiones en vez de evaluar las personas, sería más conveniente para las empresas revisar el diseño de los puestos de trabajo y funciones laborales; ubicando luego a las personas con los perfiles más adecuados para cada posición. Por ejemplo, en vez de enfocarse en los posibles deteriores cognitivos de los adultos mayores, las organizaciones se beneficiarían aprovechando sus servicios en posiciones que exijan un alto componente de inteligencias cristalizadas.

Otro elemento fundamental consiste en involucrar a los diversos grupos de interés, o 'stakeholders'; pues tal como sugieren Patrickson y Hartmann, "las medidas de desempeño solamente pueden ser desarrolladas una vez que todos los involucrados se pongan de acuerdo en lo que constituye una buena performance" (1995), así se comprendería mejor el por qué de las divergencias de medición y se avanzaría hacia la concepción de puestos de trabajo donde confluyan las actitudes y capacidades de las personas que eventualmente los ocuparían.

Si bien las organizaciones no son necesariamente las causantes del descenso de desempeño de sus empleados; ellas siempre pueden ser parte de la solución. Permitiendo miradas de largo plazo, ampliando las evaluaciones más allá de análisis de productividad, y mirando la salud ocupacional, la calidad de las relaciones de trabajo, la continuidad generacional... en fin, en las empresas radica la decisión que los cargos coincidan con las preferencias y capacidades de las personas, lo cual además de aumentar las posibilidades de justicia en las evaluaciones mejoraría la totalidad del desempeño organizacional.

\section{Motivación}

La 'buena' administración del personal en edad madura es uno de los retos más significativos para los gerentes de recursos humanos. Las expectativas de cada grupo de edad son diferentes y merecen ser tenidas en cuenta; más aún en la actual coyuntura económica, las contribuciones potenciales y los riesgos de escasez pueden generar efectos de gran magnitud en las organizaciones.

La suerte de las organizaciones depende así en gran medida de la motivación de sus empleados, la cual es afectada por la receptividad hacia los cambios provocados por la edad y por 
la factibilidad de desarrollar buenas carreras, evitando los estancamientos o 'plateau' indeseados. Por ejemplo, un estudio acerca de las experiencias y opiniones de 721 personas se mostraba como la edad y el estatus administrativo afectan significativamente la satisfacción laboral, el compromiso organizacional, y la administración (Smith \& Hoy, 1992). Por lo tanto, los autores del estudio resaltan la conveniencia de 'motivar' a los empleados a aprender nuevas habilidades y a asumir una actitud más positiva hacia el trabajo.

Analizando las vicisitudes de la carrera, Benson Rosen y Thomas Jerdee muestran como la creación de 'senderos de carrera' (profesional) ayuda a la retención de aquellos 'seniors' con competencias deseables para la empresa; así como para estimular a todos a evitar la obsolescencia de actividades (1990). Según las investigaciones de Rosen y Jerdee los 'plateau' en las carreras profesionales se explican por la baja motivación y la ausencia de sistemas administrativos que impulsen las carreras organizacionales, así como a los cambios bruscos en las responsabilidades de los puestos de trabajo.

Cómo podemos observar, la motivación de los empleados de edad, sugiere una sensibilidad especial a sus intereses particulares, así como al tipo de tareas y contexto que enmarcan su labor. De esta forma, las condiciones culturales y el sector de empleo determinan significativamente las posibles estrategias de motivación. Estudios focales constituyen referentes impor- tantes de cara a eventuales análisis comparativos y modelos generales. Así por ejemplo, un estudio realizado en Québec examina los pormenores del envejecimiento del personal en las agencias públicas, encontrando que a medida que las personas llegan a los 50 años sus expectativas -de oportunidades, entrenamiento, reconocimiento, compensación, etc.- cambian significativamente (Saba, Guerin, \& Wils, 1998). Sus hallazgos implican la conveniencia de buscar la convergencia de las expectativas metamorfoseadas de los empleados con los requerimientos de cada puesto de trabajo. ¿Pero como avanzar hacia tal convergencia?

En un estudio con gran legitimidad académica acerca de la motivación en las organizaciones, lo encontramos en el Academy of Management Review, donde Ruth Kanfer y Phillip Ackerman proponen un "marco de referencia para entender como los cambios relacionados con la edad afectan la motivación en el trabajo . Los investigadores identifican también cuatro trayectorias de cambio intraindividual (perdida, ganancia, reorganización e intercambio) que pueden rastrearse en variables tales como las habilidades cognitivas, la personalidad, los afectos, valores y los intereses vocacionales; y que en su conjunto y conjugación afectan la efectividad de las estrategias motivacionales con los adultos mayores (p. 443445).

De este artículo además merece destacarse la diferenciación (que rescatan de investigadores anteriores), de dos tipos fundamentales de habilidades in- 
telectuales: 1) habilidades fluidas, llamadas Gf, y 2) habilidades cristalizadas, llamadas Gc. Las primeras -Gf- se asocian con la memoria de trabajo, el razonamiento abstracto, la atención y el procesamiento de información nueva. Las segundas - Gc- representan amplios aspectos del conocimiento formal o experimental. Las evidencias discutidas por los autores señalan que las Gf tienden a disminuir con el paso de los años, mientras que existe consenso respecto a que las Gc tieden a aumentar gracias al paso del tiempo (Kanfer \& Ackerman, 2004: 443).

Los planteamientos anteriores sugieren la necesidad de revaluar los programas de entrenamiento y las teorías motivacionales; ir más allá de los estereotipos y descubrir (respetando y cultivando) las particularidades de cada etapa de la vida; con sus aportes y limitaciones singulares.

\section{Entrenamiento}

El entrenamiento es un componente crítico de los esfuerzos de retención de los empleados de mayor edad (Armstrong-Stassen \& Templer, 2005). En relación con el envejecimiento encontramos estudios de tres tipos: 1) el entrenamiento de todo tipo de personal prestador de servicios para atender mejor a los viejos, 2) el entrenamiento dirigido a los viejos, para mantenerlos útiles como fuerza laboral, 3) el entrenamiento ofrecido por las personas de más experiencia, para asegurar la transmisión de saberes dentro de la organización.
1) Motivados por razones tan diversas como la responsabilidad legal/ social del cargo, oportunidades de mercadeo o perspectivas financieras; muchas empresas procuran entrenar mejor a sus empleados de tal forma que puedan prestar mejores servicios a la población de adultos mayores.

Casos significativos ocurren en el sector salud, donde la presencia masiva de las personas de edad debe motivar a todo el personal involucrado a adoptar prácticas de servicio dirigidas a satisfacer sus requerimientos particulares. Por ejemplo Lach \& Renna, (1991) explican como un hospital implementó un 'task force approach' para mejorar la atención a sus pacientes de edad; entre sus logros se destacan seminarios de sensibilización para desmitificar los estereotipos acerca de la vejez, conocer los obstáculos reales en la prestación del servicio, mejorar el entrenamiento 'gerontológico' de las enfermeras.

2) El entrenamiento dirigido a mantener las competencias de los viejos aun debe desarrollarse en múltiples sentidos; en particular encontramos que el énfasis se ha hecho en permitir los ajustes a nuevas tecnologías.

Un buen ejemplo lo encontramos en el Journal of High Technologies Management Research, que evidencia como las firmas que participan de contextos cambiantes, (e.g. las que desarrollan o aplican nuevas tecnologías) son aquellas cuyo personal debe reentrenarse con más intensidad y frecuencia; y 
que curiosamente los resultados del entrenamiento dependen más de las expectativas (e.g. de duración en la empresa) que de la edad de las personas (Larwood, Ruben, Popoff, \& Judson, 1997).

Respecto al entrenamiento, también conviene recordar las ideas de Peter Warr, respecto a que los tiempos más lentos de aprendizaje de los viejos se explican por la dificultad de 'desaprender' marcos de referencia previos (Patrickson \& Hartmann, 1995); es decir al peso de la institucionalización de constumbres, y no por limitaciones cognitivas. Esta situación, nos indica la necesidad de repensar los programas de entrenamiento de tal forma que se prevea la compatibilidad con referentes institucionales. Así, los empleados de más edad no sólo podrán aprender más, sino que podrán construir nuevas síntesis de conocimiento y transmitirlas al resto de la organización.

3) Mentoring y Coaching

"Whatever the shape of the program, a host of studies show that positive mentoring experiences increase productivity, employee retention and job satisfaction. But mentoring efforts are faltering on several fronts..."

(Zaslow, 2003)

Dentro de los programas de entrenamiento ofrecidos para aprovechar el saber de las personas más experimentadas y asegurar su transmisión, aparecen diversos programas de coaching y mentoring. Concebidos para evitar las discontinuidades organizacionales y las eventuales perdidas de conocimiento (derivadas del salto generacional que se da con el retiro de los baby-boomers), también pueden ser fuente de enriquecimiento para los involucrados.

Las definiciones de ambos presentan ambigüedades respecto a su contenido y alcance; en ocasiones se utilizan indiscriminadamente en distintos contextos. Como lo sustenta Dresina Shewbridge "algunos autores no distinguen los términos, mientras que otros si hacen la distinción; cualquier opción genera problemas, dependiendo de las experiencias previas del individuo [...] la impresión general es que mientras el coaching se enfoca en el logro de objetivos específicos y mesurables el mentoring implica una aproximación más amplia y holística hacia el desarrollo integral del individuo" (Shewbridge, 2002).

Cómo la discusión semántica desborda los alcances de este trabajo, pasemos a revisar la forma en que ambos pueden usarse para abordar el problema del envejecimiento del personal.

Una de las más recientes referencias que pudimos encontrar, es un breve pero contundente artículo en el que William Seidman y Michael McCauley examinan las ideas convencionales acerca del conocimiento que se busca adquirir a partir de los seniors y sugieren formas de aclarar las imperfecciones que estos contienen (2005).

Alrededor del concepto central de Retiring Knowledge Workers (RKW); los autores recomiendan que el conocimiento de los RKWs se debe aprehender permanentemente, mediante proce- 
sos que implican comunicación y retroalimentación continua involucrando a los eventuales sucesores (en vez de reservar la transferencia para momentos previos al retiro); buscando su aplicación inmediata (en vez de su almacenamiento). Más importante aún, Seidman y McCauley nos indican que se debe intentar recuperar todo tipo de conocimiento; los esfuerzos deben enfocarse en captar el conocimiento tácito de quiénes se obtienen logros excepcionales a partir de los saberes críticos para la organización (2005).

Cómo podemos darnos cuenta, no necesariamente todos los empleados de edad se erigen como 'mentores' 'productivos' para las organizaciones. Las competencias críticas y conocimientos tácitos, son las más apetecidas por la organización, sin embargo, no todas las personas son aptas o están dispuestas a servir como mentores.

Por ejemplo, un estudio publicado en 2005 por Career Development International explora la incidencia del compromiso afectivo, las aspiraciones de carrera y las actividades de red; en las prácticas de mentores (Emmerik, Baugh, \& Euwema, 2005). Desafortunadamente para la empresa y los eventuales pupilos, los resultados de este estudio muestran que la principal motivación de los mentores voluntarios es su propia carrera profesional. Quienes en vez de una actitud de servicio y enseñanza sincera, adoptan una perspectiva instrumental de la actividad para poder ascender creando un grupo de personas leales a su causa.
Al explorar las dimensiones éticas del mentoring Business Ethics Quaterly expone ciertos comportamientos patológicos de los mentores y los riesgos de abuso que corren sus 'protegidos'; realidades distantes de la imagen virtuosa y deseable para asegurar la continuidad del capital humano y del conocimiento en las organizaciones (Moberg \& Velasquez, 2004).

Aunque es fácil percatarse de que ciertos conceptos nobles pueden convertirse en instrumentales modas de negocios; nuestra actitud al respecto debe ser constructiva. Al evocar la imagen del ideal griego, inspirado por Mentor, el protector de Telémaco, conviene contemplar diversas formas de valorar la contribución de los viejos a las organizaciones.

Algunos autores sugieren conectar la evaluación de los empleados con la planeación del relevo generacional; concretamente contribuyendo a programas de mentoring, diversidad laboral, desarrollo de carrera, entrenamiento y planificación del retiro (Soonhee, 2003).

Otros examinan las contribuciones positivas del mentoring al desarrollo de los individuos y los equipos (Post, 2003; Pullins \& Fine, 2002; Richard, Taylor, Barnett, \& Nesbit, 2022; Ritchie \& Genoni, 2002; Rosser, 2005; Scandura \& Williams, 2004), mostrando entre otros los beneficios para el mentor que asume su papel con honestidad, el pupilo receptivo, y la organización que es transparente y no instrumental en la implementación de los programas de coaching y mentoring. 
Un trabajo particularmente interesante es el publicado en Group and Organization Management, donde se examina el role de la edad en las relaciones de mentoring. (Finkelstein, Allen, \& Rhoton, 2003). Los temas tratados ilustran ciertas sutilezas del contexto universitario, resaltando variables que pueden nutrir diversas perspectivas de la investigación, tales como: percepciones de competencia, respeto, afinidad y confort interpersonal.

En resumen, podemos afirmar que las actividades de mentoring y coaching, constituyen un importante nicho donde las personas de más edad pueden contribuir significativamente a sus organizaciones. Es importante reconocer las limitaciones y riesgos del programa, evitar la formalización excesiva; pero sobretodo reconocer los aportes de los involucrados. Lo ideal sería permitir las contribuciones espontáneas y mutuamente benéficas para la organización y los participantes.

Como lo documenta Cynthia Golden en EDUCAUSE Review: muchos profesionales se involucran en distintas formas de mentoring sin percatarse del aporte que hacen a la organización (2002). Tal como ella lo afirma, los esfuerzos consisten entonces en que aquellas personas que han alcanzado niveles más altos de competencia en su campo respectivo, piensen como pueden contribuir a su oficio (Golden, 2002). En este sentido, rescatar la perspectiva individual emerge como una condición fundamental para poder construir organizaciones sanas.

\section{La perspectiva individual:}

Aunque la literatura en administración suele privilegiar los intereses de las empresas (procurando descubrir regularidades en el comportamiento de grupos e individuos que puedan servir a sus intereses), también hemos hallado varios estudios que adoptan prioritariamente el punto de vista de los individuos. A continuación evocamos algunos de estos ejemplos.

La publicación 'científica' Management Review nos presenta un elocuente artículo acerca de la 'generación sándwich' donde se pueden ver las distintas dimensiones (cuantitativa y cualitativa) y niveles (macrosocial, mesoorganizacional y micro-individual); perfilando problemáticas de investigación que aún falta profundizar (Durity, 1991). Así, además de presentar los costos macroeconómicos y organizacionales que implican el ausentismo y otros traumatismos laborales para las personas que cuidan a sus padres mayores e hijos; la autora resalta que los agregados estadísticos no revelan los desafíos reales que a nivel psicológico enfrentan las personas, denunciando además el poco respaldo que reciben de sus empleadores.

$\mathrm{Si}$ bien las personas que padecen el 'sandwich' generacional son las más afectadas, también es cierto que tensiones respecto al envejecimiento suelen afectar a todas las personas a medida que alcanzan 'el punto medio' de sus carreras. Manfred F.R. Kets de Vries, en el European Management 
Journal nos muestra diversas dificultades sicológicas -tales como temor frente a la vejez, disminución de la potencia física, competencia generacional, etc.- que provocan preocupación $\mathrm{y}$ angustia. De Vries también presenta diversas propuestas para enfrentar las mencionadas crisis de la edad avanzada (Kets De Vries, 1994).

Además de los desafíos de la jubilación para las empresas, debemos preguntarnos ¿en qué consiste la experiencia para los individuos? Una investigación efectuada en Inglaterra nos sirve de referente para entender las percepciones individuales, a saber: Graham Nelly, al consultar a 225 administradores británicos (a punto de retirarse, entre 40 y 64 años) descubrió que ellos conciben la jubilación como un proceso transicional, donde se operan aumentos y disminuciones en contraste con la vida laboral (Kelly, 1981). De este estudio merece destacarse la percepción de 'proceso' en vez de ruptura, y el hecho de que se escucha directamente a los individuos, haciendo eco a sus múltiples expectativas de cambio, temores y esperanzas.

Entre los estudios que privilegian la perspectiva del individuo, encontramos aquellos que ayudan a sustituir los estereotipos por descripciones más cercanas de la realidad, acercando los hallazgos de otras ciencias a las disciplinas administrativas. En ese sentido resulta plausible la divulgación de los trabajos del sicólogo de Harvard Douglas H. Powell, en el Journal of Management Consulting, quien encuentra que en el plano cognitivo, una proporción significativa de hombres y mujeres obtienen puntajes similares o superiores o los jóvenes (es decir, en las habilidades Cf que presentamos en la sección acerca de la motivación), así como promoviendo algunas tácticas para mantener el potencial cognitivo (Bushko \& Raynor, 1999).

Leonie Still y Wendy Timms (1998) también nos muestran una forma de resaltar la dimensión personal del envejecimiento al analizar las decisiones y expectativas de vida de 33 mujeres profesionales. Mujeres que, estando alrededor de sus 50 años, son protagonistas y testigos de las encrucijadas personales que enfrentan las personas potencialmente longevas, con las barreras y expectativas sui generis que a menudo deben enfrentar las mujeres. Aunque su investigación se efectuó en Australia, las temáticas discutidas y las metodologías implementadas sugieren referentes para eventuales investigaciones comparativas.

Terminemos esta sección acerca de las perspectivas personales, abordando el tema sensible, pero omnipresente de la muerte; respecto al cual se podrían escribir sin número de reflexiones de gran profundidad. Digamos por lo pronto, que la muerte es un tema que a pesar de ser cercano a la vejez, es raramente mencionado en la literatura administrativa de los RRHH -aunque por su relevancia ontológica merecería múltiples reflexiones-.

Los mecanismos de negación se constatan no sólo a nivel individual y organizacional -como lo advierte Gareth Morgan, en su capítulo acerca de 
las cárceles psíquicas- (Morgan, 1997), sino también en gran parte de las 'ciencias administrativas'.

Al respecto encontramos una reflexión profunda en el pensamiento de Burkard Sievers quien denuncia la manera patológica en que la cultura occidental niega la muerte y la separa de la vida, al punto que "la lucha por la inmortalidad se haya convertido en la dinámica central de las empresas contemporáneas de trabajo (Sievers, 1994: 57). Tal cómo lo podemos intuir, la discusión podría ser 'eternamente' fascinante, mucho más allá del propósito del presente texto; pospongámosla entonces tratando de conservar en mente las palabras de Sievers: "Byignoring death we have lost our frame of life" (p:56).

Para no desviarnos del tema, mencionemos el caso reportado en el $\mathrm{Jo}_{\mathrm{O}}$ urnal of Management Consulting, acerca del fundador de una compañía que preocupado por su muerte eventual y el futuro de la empresa, evade los temas relacionados con los procesos de sucesión o las decisiones que escapan a su control (Horringa, 1993).

Aún más dramático puede resultar el 'miedo a la nada' que puede suscitar una forma de ansiedad discutida por Manfred Kets de Vries (1991), provocando "la necesidad de vencer la muerte dejando algún tipo de legado", que a menudo acarrea la adicción al poder como una forma de reducir el temor de que el legado pueda ser destruido.

Todas las personas, sin importar la posición que ocupen dentro de la organización, necesitan prepararse para la transición, de tal forma que esta pue- da ser personalmente enriquecedora y socialmente útil; de tal forma que puedan constituirse legados personales que respeten la memoria y aseguren la continuidad de la empresa, pero también la salud de la persona.

Considerar la perspectiva del individuo, ponerse en su lugar, constituye un mecanismo inigualable para reintroducir la humanidad en nuestras organizaciones, y considerando que todos somos viejos en potencia, podemos desde ya hacer esfuerzos para reducir la 'ruptura entre las empresas y los hombres', concibiendo estrategias para conectar el trabajo y el retiro, con las otras esferas de la vida. En este sentido, nociones como la de bridge employment despunta como un prometedor hito de esperanza.

\section{Bridge employment}

En el esfuerzo por reconocer el potencial de cada etapa de la vida del individuo, y de facilitar su proyección social y organizacional, merece destacarse la noción de bridge employment' o 'empleo puente,' que puede definirse como 'aquel que ocurre después de que una persona se retira de su trabajo a tiempo completo y hasta antes de que se retire definitivamente de la fuerza laboral (Kim \& Feldman, 2000).

La noción de empleo puente revela una forma novedosa de aproximación de las ciencias administrativas a las realidades de la vejez; susceptible de ser explorada a nivel individual (e.g. cuales son las motivaciones y efectos en cada persona); organizacional (e.g. para re- 
velar los tipos de uso y los intereses detrás de ellos); macroeconómico (e.g. para evaluar su efecto en los niveles de salario, desempleo de otras franjas de empleados) y social (e.g. integración social, trasmisión de saberes, etc.).

Como Seongsu Kim y Daniel Feldman sostienen en su publicación en el Academy of Management Journal, la modalidad 'bridge' de empleo ha recibido muy poca atención por parte de las ciencias sociales, especialmente respecto a los factores de carácter no económico. Para contribuir a suplir ese déficit ellos realizan un estudio ingenioso que abre prometedoras vetas de investigación para explorar lo que sucede más allá del retiro laboral. Kim y Feldman examinan los factores que influencian y limitan las actitudes de las personas a buscar continuidad mediante el bridge employment. Encontrando correlaciones positivas con el nivel de salud, la estabilidad/respaldo organizacional, la existencia de cónyuge laboralmente activa(o) y de hijos dependientes; y correlaciones negativas con la edad y los salarios.

Todas las anteriores variables evocan la importancia de adoptar una perspectiva individual al analizar el problema del envejecimiento, de reinstaurar el respeto por el hombre, y de evitar las discriminaciones por su edad.

\section{La preocupación entorno a la discriminación por la edad}

Until employers can be convinced that it is not in their best interest to discharge older workers simply becau- se of their age, it is essential for our nation to maintain strong laws and policies against age discrimination. Age discrimination in the workplace can not be tolerated. [...] it, should be reminded that aging is a part of life. Each and every person will be part of the "older" work force eventually and will have to deal with age discrimination in the work place unless steps are taken now to eliminate it. (Engleman \& Kleiner, 1998)

La discriminación y los prejuicios por la edad constituyen una patología social que desborda las fronteras geográficas e históricas. En las actividades organizacionales se advierte la vulnerabilidad de los viejos, quienes son victimas de una reducción de oportunidades, las cuales se acentúan con el advenimiento de cambios técnicos y organizacionales (Barnum, Liden, \& DiTomaso, 1995).

El reto es aún mayor en la época contemporánea, donde los pronósticos indican que las fuerzas laborales que envejecen están compuestas por un número creciente de mujeres y comunidades étnicas que han sido tradicionalmente discriminadas. Desafortunadamente, la desigualdad respecto a los hombres blancos aumenta con la edad, tal como se desprende del análisis de remuneración reportado en el Academy of Management Journal (Barnum et al., 1995).

Por eso todos los esfuerzos de denuncia constituyen aportes plausibles dentro del área de RRHH. Por ejemplo, Phil Lyon e Ian Glover denuncian el silencio y la negación respecto al 
'ageism', y el hecho de que el campo de los RH haya reducido la problemática de la discriminación a los tratos peyorativos en la publicidad y a los casos más visibles de marginación en la contratación (1998).

Una contribución significativa de Lyon y Glover es su demostración que para "justificar la discriminación, los empleadores han atribuido (a los viejos) deficiencias generalizadas en términos de habilidades, actitudes y energía, aumentando los estereotipos culturales acerca del proceso de envejecimiento (Lyon \& Glover, 1998).' Efectivamente, las imágenes sociales y narrativas históricas respecto a la vejez, son una fuente de prejuicios que afectan las relaciones entre las distintas generaciones, penetrando las múltiples esferas de la sociedad.

El problema consiste en que la patológica situación, aunque omnipresente, es mucho menos visible de lo que debería ser, erigiendo una especie de tabú ${ }^{2}$ y la negación de una problemática aunque esta en todas partes, con frecuencia se ignora en todas.

Circunscribiendo la discusión al ámbito organizacional, constatamos que la discriminación hacia los viejos, efectivamente fragua realidades para- dójicas que a menudo perjudican la pretensión de los mismos logros económicos que la impulsan. Por ejemplo, Emma Brockes publicaba en The British Journal of Administrative Management los excesos del 'super ageism' que despuntaba a finales del siglo pasado; y bajo el cual se sentenciaba draconianamente que las personas alcanzaban el máximo desempeño hacia los 26 años, a los cuales seguía el ineluctable declive. Esta actitud discriminatoria genera inconvenientes posteriores para el bienestar organizacional, derivados de la perdida de memoria corporativa y de la incapacidad de los 'jovenes' administradores de vincular personas de mayor edad (Brockes, 1999).

La discriminación basada en la edad, puede ser también la consecuencia de la implementación de racionalidades superfluas que buscan "deshacerse de los empleados más viejos y costosos y encontrar reemplazos más jóvenes y baratos (Capowski, 1994). Cómo vemos, la cuestión no sólo es de imagen, sino también de lógicas y dinámicas que buscan la maximización 'monetaria' en el corto plazo, ignorando la sostenibilidad en el largo plazo ${ }^{3}$. Este tipo de prácticas se evidencia en

2 Tabú, que nos recuerda la explicación Freudiana acerca del parricidio en la horda imaginaria y la eventual fundación de la civilización, con la consecuente 'idealización/ totemización' del padre inmolado -hipótesis algo descontextualizada pero que bien podría ayudar a explicar la brecha entre los comportamientos reales y las actitudes simbólicas hacia los viejos.

3 Incluso, ese mismo tipo de mentalidad motiva ciertas decisiones de reemplazar empleados de cualquier edad, por otros mayores que gracias a su tiempo disponible, necesidad de recursos o por el simple hecho de sentirse útiles trabajan por salarios 
los sesgos presentes en las prácticas de downsizing que se 'encarnizaban' en los empleados más antiguos; introduciendo iniquidad e ignorando las contribuciones positivas de los adultos mayores (Patrickson \& Hartmann, 1995).

Gracias a la visibilidad de problemáticas relacionadas con el envejecimiento, así como al aprendizaje de los errores cometidos en el pasado muchas organizaciones (y la misma sociedad) comienzan a apreciar las contribuciones potenciales de los adultos mayores. Este es un motivo de esperanza... de que todas las personas reduzcamos la iniquidad y re-descubramos el valor (y no sólo la utilidad) de los ancianos; bien sea que estén laboralmente activos o que se encuentren en retiro.

Mientras tanto, para evitar la discriminación, conviene seguir el consejo de Doreena Engleman y Brian H Kleiner quienes, después de examinar las prácticas empresariales norteamericanas, sostienen con vehemencia la necesidad de "mantener leyes fuertes y políticas contra la discriminación, [...], debe recordarse que envejecer es una parte de la vida" y que puesto que todos seremos parte de una fuerza laboral envejecida, a todos nos conviene esforzarnos por reducir la discriminación (Engleman \& Kleiner, 1998).

\section{Lógicas}

Al examinar las aproximaciones del conocimiento administrativo acerca del envejecimiento, podemos percatamos de la existencia de diversas lógicas que permean su construcción y sus alcances: Temores respecto a la disminución del desempeño; aumento del costo de los salarios; perdida de información clave para las organizaciones... estos son algunos de los motivos que estimulan el estudio de problemáticas afines.

Para comprender los saberes disponibles y eventualmente elucidar caminos de acción y de investigación, a continuación proponemos una interpretación de las lógicas que aparecen como subyacentes.

La reflexión acerca de las lógicas la articulamos alrededor de cinco preguntas, a saber:

a) ¿Predomina la perspectiva individual o colectiva?

b) ¿Cuáles son las aproximaciones metodológicas utilizadas?

c) ¿Qué dimensiones filosóficas sobresalen en la literatura existente? (i.e. ontológicas, axiológicas, epistemológicas o praxeológicas?)

d) ¿Cuáles de las funciones sociales suscitan mayor interés (i.e. dentro de las identificadas por George Dumezil: fecundidad, seguridad, soberanía)?

inferiores. Lo más triste, es que este desplazamiento de sectores laborales, por puestos marginales y mal remunerados; con frecuencia se presenta luego a la opinión pública como creación de empleos. 
e) ¿Qué tipo de racionalidad (según las categorías de Weber) predomina en el análisis y conocimientos existentes?

\section{a) Perspectiva: ¿individual o colectiva?}

Podríamos decir que en el campo de RRHH ambas perspectivas son tenidas en cuenta: Hay conciencia del impacto agregado generado por la transición generacional (especialmente visible con los baby-boomers); como también reconocimiento del impacto concreto que la jubilación de ciertos individuos (sobre todo los dirigentes) provoca en cada organización.

Sin embargo, observamos que en general se concede gran valor a las estadísticas y agregados, opacando un poco las voces y dimensión 'humanas' de todas y 'cada' una de las personas participantes. Esto puede ser el resultado del tipo de formato preferido por las 'publicaciones científicas', pero también es la consecuencia de privilegiar las perspectivas de la empresa sobre las necesidades de cada individuo. Aunque alguien podría deslegitimar esta queja argumentando que "la responsabilidad de las empresas es generar utilidades" (tal como lo argumenta el premio Nobel de economía Milton Friedman) y preocuparse por el bienestar de las personas; uno podría objetar tal pretensión y re-situar el debate desde vectores e interrogantes genuinamente humanos.

Incluso cuando se privilegia la mirada individual es preciso estar atentos a posibles riesgos derivados de sobrecargar de responsabilidades a las personas, y convertirlas en responsables de sostener el sistema; tal como ocurre con ciertas prácticas de coaching y mentoring, a través de las cuales las organizaciones se desprenden de su responsabilidad mientras depositan gran peso en los individuos, sin protegerlos de los peligros emocionales y sociales inherentes. Preguntémonos por ejemplo, acerca de las causas del estrés en las personas que practican alguna profesión, y si frente al mal diseño del sistema son los individuos quienes deban pagar las consecuencias

\section{b) Aproximación:}

¿cualitativa o cuantitativa?

En el campo de los RRHH hemos observado la doble presencia de aproximaciones cualitativas y cuantitativas. A veces de manera simultánea, otras aislada, pero siempre potencialmente complementarias.

$\mathrm{Al}$ abordar la problemática de los impactos sobre los costos, la producción o medidas de desempeño se percibe la tendencia cuantitativa. Para captar tendencias macro, los académicos proponen encuestas y cuestionarios a los ejecutivos de RRHH, y luego agregan los datos proyectando patrones de comportamiento y prediciendo fuerzas del entorno. Al abordar a los individuos se recurre a técnicas cualitativas, como entrevistas a profundidad y semi-estructuradas, relatos de vida y discusiones grupales.

En esta instancia lo más destacable es la necesidad de avanzar en la 
profundización y complementarización de ambas aproximaciones. Aunque la realidad humana es irreductible a lo cuantitativo, esta perspectiva nos ofrece un lenguaje que las organizaciones comprenden y que nos puede ayudar a identificar las acciones prioritarias a seguir (obviamente, si se hacen las preguntas adecuadas).

\section{c) Dimensiones filosóficas}

En términos generales se observa que tanto los académicos, como los departamentos empresariales de RRHH, se ocupan prioritariamente por rescatar ciertos 'conocimientos y habilidades' de las personas que parten al retiro. Las referencias ontológicas son prácticamente inexistentes, $\mathrm{y}$ cuando se habla de valores, la alusión es más enfocada a la 'cultura corporativa' que a una verdadera contribución axiológica.

Mientras que la literatura gerontológica habla de 'buena vejez', en las ciencias administrativas se insiste en la noción de 'vejez productiva' -durante el periodo de actividad laboral de la persona-, o de 'vejez exitosa' (successful aging), con dinero y sin grandes preocupaciones -durante la jubilación-.

La orientación excesiva hacia las prácticas, habilidades y conocimientos del oficio es una consecuencia directa de la preponderancia de las necesidades de la empresa (y la economía en general) sobre las necesidades del ser humano, como especie y como individuo. El lenguaje ofrece una evidencia clara: por lo general se habla más de desempeño que de calidad de vida; de costos para la empresa que de enriquecimiento personal.

Afortunadamente a medida que avanzan ciertos conocimientos se reducen los prejuicios y se amplía el espectro de aspectos a considerar en la evaluación. Por ejemplo, en cuanto a las teorías de la motivación hay más respeto por el ser humano y se aboga por buscar convergencias entre sus expectativas y las de la organización (y no la imposición de estas últimas).

Los cuestionamientos son cada vez más frecuentes respecto a la necesidad de crear condiciones de tiempo y lugar para que 'el hombre' pueda desplegarse a plenitud, según su edad y motivaciones internas. Afortunadamente, en vez de forzar la adaptación de las personas a los puestos de trabajo, las empresas parecen ser progresivamente receptivas a la idea de un diseño a partir de las personas (en gran medida porque el envejecimiento del personal no les permite muchas otras alternativas).

\section{d) Tri-funcionalidad}

Una interpretación a partir de la teoría de la trifuncionalidad -formulada por el historiador frances Georges Dumezil (1958)-, nos conduce a pensar que las preocupaciones predominantes gravitan alrededor de la producción y la creación; con ciertos interrogantes respecto al gobierno y al interés general.

Las discontinuidades que a nivel laboral pueden acarrear las transformaciones demográficas (olas de jubi- 
laciones, escasez de reemplazos competentes, etc.), hacen que las empresas se preocupen fundamentalmente por los impactos que a nivel de la 'fecundidad' de sus operaciones pueda tener la recomposición más o menos súbita de la fuerza laboral.

Temores respecto al aumento de los costos de seguridad social (por jubilación o por salud), perdida de memoria colectiva, de conocimientos y habilidades críticas; disminución de la productividad... son algunos de los factores de intranquilidad que al mismo tiempo opacan las contribuciones potenciales que los viejos están en capacidad de efectuar.

En ese sentido es reconfortante pensar que las conceptualizaciones teóricas (e.g. habilidades cristalizadas, inteligencias múltiples de Howard Gardner) permiten hacer más visible los aportes potenciales de los adultos mayores. Algunas organizaciones hacen eco al arquetipo positivo de la vejez, y perciben en sus miembros de más edad las fuentes de prudencia y sabiduría que puedan orientar e inspirar al resto de sus miembros.

Un gran avance del conocimiento en RRHH es sugerir la posibilidad de que los viejos pueden coadyuvar 'al gobierno de los hombres', para no desgastarlos así en el 'manejo de las cosas'. En la medida que se reequilibre la tensión y complementariedad necesaria entre las tres funciones podremos advenir a una sociedad mejor, con organizaciones más sensatas e individuos más felices. e) Racionalidad (substantiva vs. procedimental/ instrumental)

Al escudriñar la literatura de RRHH encontramos que tanto la racionalidad instrumental/procedimental como la sustantiva son utilizadas por distintos tipos de investigaciones.

La racionalidad instrumental persiste en los análisis de costos, así como en las teorías de motivación y evaluaciones de desempeño que perfeccionan el 'know how' ignorando el 'know why' y sus impactos sobre el ser humano. Afortunadamente, como la continuidad misma de muchas organizaciones depende de los aportes de una fuerza laboral que envejece, cada vez se concede más espacio a las reflexiones substantivas acerca de las personas que sostienen la organización, $\mathrm{y}$ que no son fácilmente reemplazables, ni en número ni en calidad.

Aunque aún falta mucho por lograr que la racionalidad substantiva domine, es reconfortante observar que a medida que pasan los años, y ante la llegada inminente de generaciones mejor educadas y con mayor poder electoral y económico; cada vez más se otorga atención a sus preocupaciones genuinas.

\section{Conclusión}

Al examinar las distintas formas en qué las ciencias administrativas han abordado el envejecimiento del personal, encontramos que el área de recursos humanos, tanto como campo aca- 
démico y como departamento activo dentro de las organizaciones es una de las disciplinas más receptivas al fenómeno del envejecimiento de la población. Sus miradas conjugan enfoques cualitativos y cuantitativos, se preocupan por distintas dimensiones del ser humano (aunque no siempre de manera equilibrada) y procuran -al menos en teoría- buscar la convergencia entre los intereses de los individuos y las necesidades de la empresa.

No obstante los avances de los últimos años, aún falta profundizar en la implementación del imperativo kantiano de tratar al ser humano como 'fin y nunca como medio'; configurando organizaciones que se conciban teniendo a la humanidad como punto de partida y de llegada; como razón sustancial que anima la acción.

Las realidades derivadas del fenómeno humano de envejecimiento, a nivel individual y colectivo, permiten y exigen que nuevos ejes de reflexión sean activados. ¿Cuáles son los aportes potenciales de los más viejos?, ¿Cuál debe ser la responsabilidad y solidaridad intergeneracional?, ¿Cómo conciliar las necesidades individuales con las dinámicas sociales?; los interrogantes son múltiples, por ahora digamos que un primer aprendizaje para valorar a ' $l o s$ viejos' dentro de la empresa consiste en evitar los discursos en términos de disyuntivas excluyentes.

Al pensar en la vejez, solemos asociarla a imágenes diametralmente opuestas; sabiduría vs. locura; perdida de autonomía vs. libertad última. El arquetipo intangible nos aleja del ser humano concreto, sobretodo en las esferas empresariales donde se fraguan falsos dilemas entre costos exagerados o ausencia debilitante; o disyuntivas extremas, donde se pretende clasificar a los viejos en antípodas excluyentes, como carga o como fuerza.

A pesar de la distinción útil entre Gf y Gc (habilidades intelectuales fluidas y cristalizadas) conviene precisar que las observaciones respecto a nuestra especie no deben circunscribirse a sus quehaceres, pues aunque homo faber, somos esencialmente home sapiens sapiens. Por lo tanto, en el contexto de las organizaciones socio-económicas-empresariales, debe concebirse al ser humano no sólo en términos de lo que puede hacer, sino también de los conocimientos que es capaz de generar. Desde este tipo de concepciones las ciencias administrativas pueden descubrir nuevas dimensiones y contribuciones derivadas del envejecimiento humano.

\section{Bibliografía}

ARMSTRONG-STASSEN, M., \& TEMPLER, A. (2005). Adapting training for older employees: The

CANADIAN RESPONSE TO AN AGING WORKFORCE. The Journal of Management Development, 24(1/2), 57.

BARNEY, S. M. (2002). A changing workforce calls for twenty-first century strategies. Journal of Healthcare Management, 47(2), 81.

BARNUM, P., LIDEN, R. C., \& DITOMASO, N. (1995). Double jeopardy for women and minorities: Pay differences with age. Academy of 
Management Journal, 38(3), 863.

BROCKES, E. (1999). Age of uncertainty. The British Journal of Administrative Management, 11.

BROOKE, L. (2003). Human resource costs and benefits of maintaining a mature-age workforce. International Journal of Manpower, 24(3), 260.

BROWNELL, J. (1994). Creating strong listening environments: A key hospitality ma. International Journal of Contemporary Hospitality Management, 6(3), 3 .

BUSHKO, D., \& RAYNOR, M. (1999). More on the aging worker: Who are they and how can they stay vital? Journal of Management Consulting, 10(3), 68.

CAMERON, K., JORGENSON, J., \& KAWECKI, C. (1993). Civil Service 2000 revisited: Old assumptions New facts and forecasts. Public Personnel Management, 22(4), 669.

CAMERON, K., \& ZAMMUTO, R. (1983). Matching Managerial Strategies to Conditions of Decline. Human Resource Management (pre-1986), 22(4), 359.

CAPOWSKI, G. (1994). Ageism: The new diversity issue. Management Review, 83(10), 10.

COATES, J. F. (1987). An Environmental Scan: Projecting Future Human Resource Trends. HR. Human Resource Planning, 10(4), 219.

CRAMPTON, S., HODGE, J., \& MISHRA, J. (1996). Transition - Ready or not: The aging of America's work force. Public Personnel Management, 25(2), 243.

DUMEZIL, G. (1958). L'idéologie tripartie des Indo-Européens. Bruxelles: Latomus.

DURITY, A. (1991). The Sandwich Generation Feels the Squeeze. Management Review, 80(12), 38.
ELLIOTT, R. H. (1995). Human resource management's role in the future aging of the. Review of Public Personnel Administration, 15(2), 5.

EMMERIK, H. V., BAUGH, S. G., \& EUWEMA, M. C. (2005). Who wants to be a mentor? An examination of attitudinal, instrumental, and social motivational components. Career Development International, 10(4), 310.

ENGLEMAN, D., \& KLEINER, B. H. (1998). Age discrimination in the workplace. Equal Opportunities International, 17(3-5), 3.

FINKELSTEIN, L. M., ALLEN, T. D., \& Rhoton, L. A. (2003). An examination of the role of age in mentoring relationships. Group \& Organization Management, 28(2), 249.

GOLDEN, C. (2002). The wise and trusted counselor. EDUCAUSE Review, $37(2), 56$.

GRIFFIN, K. (2005, September \& October). You're wiser now. AARP.

HADER, R. (2005). The golden years: Sooner than we think? Nursing Management, 36(3), 6 .

HAMMETT, J. R. (1984). The Changing Work Environment. Employment Relations Today, 11(3), 297.

HORRINGA, D. (1993). Consulting across the grave. Journal of Management Consulting, 7(3), 35 .

KANFER, R., \& ACKERMAN, P. L. (2004). AGING, ADULT DEVELOPMENT, AND WORK MOTIVATION. Academy of Management. The Academy of Management Review, 29(3), 440.

KELLY, G. M. (1981). Anticipating Retirement: The Manager's View. Journal of General Management, 7(2), 36.

KETS DE VRIES, M. F. R. (1991). Whatever Happened to the PhilosopherKing? The Leader's Addiction to 
Power. The Journal of Management Studies, 28(4), 339.

KETS DE VRIES, M. F. R. (1994). Can you manage the rest of your life? European Management Journal, 12(2), 133.

KIM, S., \& FELDMAN, D. C. (2000). Working in retirement: The antecedents of bridge employment and its consequences for quality of life in retirement. Academy of Management Journal, 43(6), 1195.

KIYONAGA, N. B. (2004). Today is the Tomorrow You Worried About Yesterday: Meeting the Challenges of a Changing Workforce. Public Personnel Management, 33(4), 357.

LACH, H. W., \& RENNA, R. S. (1991). A Task Force Approach to Managing Aging Issues. Nursing Management, 22(1), 62 .

LARWOOD, L., RUBEN, K., POPOFF, C., \& JUDSON, D. H. (1997). Aging, retirement, and interest in technological retraining: Predicting personal investment and withdrawal. Journal of High Technology Management Research, 8(2), 277.

LEE, M. Y. (1991). Changing Personnel Management Practices: A Case Study of a Japanese Firm. Journal of Small Business Management, 29(3), 83.

LYON, P., \& GLOVER, I. (1998). Divestment of investment? The contradictions of HRM in relation to older employees. Human Resource Management Journal, 8(1), 56.

McCUNE, J. C. (1990). Japan Says Sayonara to Womb-to-Tomb Management. Management Review, 79(11), 12.

MENEFEE, J. A. (1983). Implications of a Changing Work Force on Future Benefits Strategies. Risk Management, 30(10), 30.

Metz, E. J. (1984). The Missing ' $H$ ' in Strategic Planning. Managerial Plan- ning, 32(6), 19.

MOBERG, D. J., \& VELASQUEZ, M. (2004). The Ethics of Mentoring. Business Ethics Quarterly, 14(1), 95.

MORGAN, G. (1997). Images of Organization. Thousand Oaks, CA: Sage Publications.

MULLENS, A. (2005). Will other universities match U of T's retirement deal? Landmark agreement creates buzz at meeting of retired faculty associations. University Affaires, 38-39.

PATRICKSON, M., \& Hartmann, L. (1995). Australia's ageing population: Implications for human resource management. International Journal of Manpower, 16(5,6), 34.

PATRICKSON, M., \& RANZIJN, R. (2004). Bounded choices in work and retirement in Australia. Employee Relations, 26(4), 422.

PATTON, A. (1981). The Coming Promotion Slowdown. Harvard Business Review, 59(2), 46.

POST, R. (2003). You gotta have heart. Public Manager, 31(4), 51.

PULLINS, E. B., \& Fine, L. M. (2002). How the performance of mentoring activities affects the mentor's job outcomes. The Journal of Personal Selling \& Sales Management, 22(4), 259.

RABEY, G. (2005). Ageing - "Carpe diem" has no use-by date. Human Resource Management International Digest, 13(1), 3.

RAPPAPORT, A. M. (1988). Human Resources Implications Of A Changing Labor Force. Benefits Quarterly, 4(2), 65.

RICHARD, O. C., Taylor, E. C., Barnett, T., \& Nesbit, M. F. A. (2022). Procedural voice and distributive justice: Their influence on mentoring career help and other outcomes. Journal of Business Research, 55(9), 725. 
RITCHIE, A., \& Genoni, P. (2002). Group mentoring and professionalism: A programme evaluation. Library Management, 23(1/2), 68 .

ROSEN, B., \& JERDEE, T. H. (1989). Retirement Policies: Evidence of the Need for Change. Human Resource Management (1986-1998), 28(1), 87.

ROSEN, B., \& JERDEE, T. H. (1990). Middle and Late Career Problems: Causes, Consequences, and Research Needs. HR. Human Resource Planning, 13(1), 59.

ROSSER, M. H. (2005). Mentoring From the Top: CEO Perspectives. Advances in Developing Human Resources, 7(4), 527.

SABA, T., Guerin, G., \& Wils, T. (1998). Managing older professionals in public agencies in Quebec. Public Productivity \& Management Review, 22(1), 15.

SCANDURA, T. A., \& WILLIAMS, E. A. (2004). Mentoring and transformational leadership: The role of supervisory career mentoring. Journal of Vocational Behavior, 65(3), 448.

SEIDMAN, W., \& McCAULEY, M. (2005). Saving Retiring Knowledge Workers' "Secret Sauce". Performance Improvement, 44(8), 34.

SHEWBRIDGE, D. (2002). Coaching and Mentoring. Industrial and Commer- cial Training, 34(3), 123.

SIEVERS, B. (1994). Work, Death, and life Itself. New York: de Gruyter.

SMITH, P. L., \& HOY, F. (1992). Job satisfaction and commitment of older workers in small businesses. Journal of Small Business Management, 30(4), 106.

SOONHEE, K. (2003). Linking Employee Assessments to Succession Planning. Public Personnel Management, 32(4), 533.

STILL, L., \& TIMMS, W. (1998). Career barriers and the older woman manager. Women in Management Review, 13(4), 143.

WEST, J. P., \& BERMAN, E. M. (1996). A national survey: Managerial responses to an aging municipal workforce. Review of Public Personnel Administration, 16(3), 38.

WOOLRIDGE, B. (1994). Changing demographics of the workforce: Implications for the use of technology as a productivity improvement strategy. Public Productivity \& Management Review, (4), 371.

ZASLOW, B. J. (2003, Jun 5, 2003). Moving On: Don't Trust Anyone Under 30: Boomers Struggle With Their New Role as Mentors. Wall Street Journal, p. D.1. 


\title{
RESÚMENES Y ABSTRACTS
}

\section{LOS VALORES EN LAS FAMILIAS EMPRESARIAS COLOMBIANAS}

\author{
Ernesto Barrera Duque
}

\section{RESUMEN}

Este artículo, a partir de una investigación cualitativa y práctica, propone un modelo general de análisis para los valores en las dinámicas operativas de las familias empresarias colombianas. En el cuerpo del texto, se identifican los valores vigentes, estableciéndose su contenido y jerarquía, en función de los resultados del trabajo de campo.

PALABRAS CLAVE: Familia empresaria, empresa familiar, valores.

\begin{abstract}
This article, from a qualitative and practical research, proposes a general model of analysis for the dynamic values in operative the families of the Colombian industralists. In the body of the text, the effective values are identified, settling down their content and hierarchy, based on the results of the work of field.
\end{abstract}

KEY WORDS: Family entrepreneur, familiar company, values. 


\title{
ANÁLISIS DEL DISEÑO DE LOS CONTRATOS EN EL PROCESO DE SUBCONTRATACIÓN DE LAS ORGANIZACIONES PÚBLICAS, EL CASO DE EMCALI EICE
}

\author{
Henry Caicedo Asprilla
}

\begin{abstract}
RESUMEN
Éste documento utiliza las categorías de la economía institucional para evaluar el impacto que tiene sobre las organizaciones públicas el proceso de outsourcing o subcontratación. Aquí se plantea el interrogante de hasta dónde es posible alcanzar la eficiencia de una organización pública cuando el proceso de subcontratación está determinado por contratos intrínsecamente incompletos. A partir de la información de una muestra de 198 contratos que Emcali firmó con proveedores entre los años 2001 y 2004, se halló que 128 estaban mal diseñados y sólo 70 cumplían con el requisito del buen diseño económico; este hallazgo permite verificar la presunción de la teoría económica institucional que afirma que aunque los contratos estén diseñados conforme a la ley, estos presentan fallas en el diseño económico y por consiguiente la empresa experimenta costos de transacción. En este docu-
\end{abstract}

mento se evidencia que en el caso concreto de Emcali existen dos variables que clasifican los contratos entre bien y mal diseñados: el valor de la inversión y el índice de riesgo de ejecución del contrato; mientras que las variables duración del contrato, el riesgo legal, empresas en licitación, garantías y pólizas de aseguramiento, consideradas fundamentales en el diseño contractual de una transacción no cumplen con el propósito de corregir las anomalías en los contratos. En este trabajo se avanza hasta plantear que existe una conexión entre el mal diseño de los contratos y las dificultades financieras y administrativas de Emcali, que llevó a la empresa a la crisis más grande de su historia.

PALABRAS CLAVE: Organizaciones públicas, outsourcing, análisis de los contratos, economía institucional, árboles de decisión. 


\section{ABSTRACT}

This paper uses the categories of the institutional economy to evaluate the impact that has on the public organizations the process of outsourcing or subhiring. The principal question is: until where considers it is possible to reach the efficiency of a public organization when the subhiring process is determined by intrinsically incomplete contracts. From the information of a sample of 198 contracts that Emcali signed whit suppliers between years 2001 and 2004, one was that 128 badly were designed and only 70 fulfilled the requirement of the good economic design, this finding allows to verify the economic presumption of the institutional theory that it affirms that although the contracts are designed according to the law, these contracts present faults in the economic design and therefore the company experiences transaction costs. This document evidences that in the Emcali's case there are two indicator that classify contracts between good and badly designed: the value of the investment and the index of risk of execution of the contract, whereas the indicators duration of the contact, the legal risk, companies in licitation, guarantees and insurance policies, were considered fundamental in the contractual design of a transaction do not correct the anomalies of the contacts. In this paper one advances when a connection is established between the wrong design of contracts and the financial and administrative difficulties of Emcali, that led the company to the greatest crisis of its history.

KEY WORDS: Public organizations, outsourcing, analysis of contracts, institutional economy, decition trees. 


\title{
¿DESDE UNA INGENIERÍA DEL ALMA HACIA UN "MANAGEMENT" DEL ESPÍRITU? ACERCA DE LA NOCIÓN DE PSICOLOGÍA EN EL DISCURSO ADMINISTRATIVO Y ORGANIZACIONAL
}

\author{
Héctor Bermúdez
}

\section{RESUMEN}

Este ensayo busca un acercamiento a la genealogía de lo que se reconoce en la administración como las "Ciencias del Comportamiento" y una crítica a la relación entre el pensamiento administrativo y aquella corriente particular de la psicología que ha sido incorporada a éste: lo que comúnmente se conoce como la psicología industrial pero que bien podría nombrarse como el conductismo organizacional.

Cabe decirse también, que, por el trabajo de consultoría especializada en Gestión Humana del autor, y por la docencia tanto en cursos de MBA para ejecutivos, como en programas para especialistas en administración de personal, fue posible articular esa experiencia de campo, con lo inferido del análisis teórico de la revisión documental. Así entonces, este trabajo busca apoyar la línea de investigación en estudios sobre la Gestión Humana.

PALABRAS CLAVE: Administración, Conductismo Organizacional, Psicoanálisis, Psicología Industrial, Organizaciones.

\begin{abstract}
This test looks for an approach of the genealogy of which a critic is recognized in the administration like "Sciences of the Behaviour" on the other hand, the article explain the relation between the administrative thought and that particular current of the psychology that has been incorporated to this one. This challenge is commonly known like the industrial psychology but that could well name like the organizational conductism.

It is possible also to say itself, that, by the work of consultancy specialized in Human Management of the author, and by teaching as much in courses of MBA for executives, like in programs for specialists in personnel administration, it was possible to articulate that experience of field, with the inferred thing of the theoretical analysis of the documentary revision. Then, this work looks for to support the line of research in studies on the Human Management.
\end{abstract}

KEY WORDS: Administration, Organizational Conductism, Psychoanalysis, Industrial Psychology, Organizations. 


\title{
LOS DESAFÍOS DEL DISEÑO ORGANIZACIONAL E INSTITUCIONAL EN UN MEDIO AMBIENTE GLOBALIZADOR
}

\author{
José G. Vargas Hernández
}

\section{RESUMEN}

Este trabajo tiene por objetivo analizar los desafíos de diseño organizacional e institucional en un medio ambiente globalizador. Este análisis se inicia a partir de las consideraciones de los procesos de globalización económica en la emergencia de las nuevas asimetrías del poder y su impacto en los procesos de cambio organizacional, institucional, del Estado y las políticas públicas y el diseño intergubernamental. La información y el conocimiento son insumos de las comunidades de aprendizaje y para la formación de redes. Una característica de los arreglos institucionales y nuevas formas organizacionales es la creación del poder en los individuos (empowerment)

PALABRAS CLAVE: Asimetrías del poder, diseño institucional, diseño organizacional, globalización

\begin{abstract}
This work must by objective analyze the challenges of organizational and institutional design in global environment. This analysis begins from the considerations of the processes of economic globalization in the emergency of the new asymmetries of the power and its impact in the processes of organizational, institutional change, of the State and the public policies and the intergovernment design. The information and the knowledge are raw material of the learning communities for the formation of networks. A characteristic of the institutional adjustments and new organizationals forms is the creation of the power in the individuals (empowerment)
\end{abstract}

KEY WORDS: Asymmetries of the power, institutional design, organizational design, globalization. 


\title{
LA REFORMA DEL ESTADO EN COLOMBIA: ENTRE EL AJUSTE FISCAL Y FORTALECIMIENTO DE LA PARTICIPACIÓN CIUDADANA
}

\author{
Rosalía Correa Young
}

\begin{abstract}
RESUMEN
La reforma del Estado en Colombia y la descentralización, desde que se aprobó el Acto legislativo 01 de 1986, pasando por los acuerdos institucionales, plasmados en la Constitución de 1991 y referidos a esta materia, así como la normatividad actual, son analizados en este artículo a la luz de los enfoques teóricos que estimularon un nuevo modelo de Estado, a partir de la percepción respecto del agotamiento del Estado de bienestar, interventor y asistencial.

El artículo explica la dinámica en Colombia frente a dos paradigmas: uno proveniente de la sociología y la ciencia política que define la reforma desde una perspectiva policéntrica y uno proveniente de la economía neoclásica que ofrece una perspectiva mercadocéntrica. La primera en-
\end{abstract}

cuentra fortalezas para la democratización, la equidad, la superación de los desequilibrios regionales y la inclusión social. La segunda, el posicionamiento de las lógicas del mercado con la reducción, o desaparición de la intervención del Estado en aquellas funciones que los privados pueden resolver más eficientemente. Uno y otro colocan a la sociedad civil en un protagonismo activo, sea político, en su relación con el Estado; sea económico, en su relación con el mercado, pero ambos la conciben como actor de primera línea.

PALABRAS CLAVE: Reforma del Estado, Descentralización, Democratización, Paradigma policéntrico, Paradigma mercadocéntrico, Ajuste Fiscal, Participación, Privatización 


\section{ABSTRACTS}

The reform of the state in Colombia and the decentralization, since legislative Act 01 of 1986 was approved, happening through the agreements institutional, shaped in the Constitution of 1991 and referred this matter, as well as the present normatividad, are analyzed in this article to the light of the theoretical approaches that stimulated a new model of state, from the perception respect to the exhaustion of the welfare state, auditor and healthcare.

The article explains dynamics in Colombia as opposed to two paradigms: one originating of sociology and political science that the reform defines from a policentric perspective and one originating of the neoclassic economy that offers a market perspective. First it finds strengths for the democratization, the fairness, the overcoming of the regional imbalances and the social inclusion. Second, the positioning of the logics of the market with the reduction, or disappearance of the intervention of the State in those functions that the prevailed ones can solve more efficiently. Both places to the civil society in an active protagonism, is politician, in their relation with the State; be economic, in his relation with the market, but both conceive it like actor of forward edge.

KEY WORDS: The reform of the State, Decentralization, Democratization, policentric Paradigm, market Paradigm, Fiscal Adjustment, Participation, Privatization. 


\section{TEORÍA ECONÓMICA Y ESTADO DEL BIENESTAR. UNA APROXIMACIÓN}

Fernando Salazar Silva

\section{RESUMEN}

El siguiente artículo realiza un tránsito por algunas posturas dentro de las ciencias económicas para discutir el EB y presentar alternativas concretas al mismo. El propósito de Keynes en su afán de hacer legitimar la configuración y consolidación del Estado de Bienestar, se discutirá en el contexto de las particularidades de carácter teórico económico. Si bien el keynesianismo pudo concretar desde las ciencias económicas las bases para acercar la teoría a la realidad social, no entendemos por qué otras escuelas no han logrado constituirse en la base teórica del Estado de Bienestar actual.

PALABRAS ClAVE: Estado de Bienestar, Pacto keynesiano, Ciencia económica, Monetarismo, Expectativas racionales, teoría Neoclásica, Neokeynesianismo.

\begin{abstract}
The following article makes a transit by some positions within economic sciences to discuss the EB and to present concrete alternatives to he himself. Keynes's intention in its eagerness to make legitimize to the configuration and consolidation of the Welfare State, will be discussed in the context of the particularities of economic theoretical character. Although the keynesianism could make specific from economic sciences the bases to approach the theory the social reality, we do not understand so that other schools have not managed to constitute themselves in the theoretical base of the Welfare State present.

KEY WORDS:

Welfare State, Keynesian Accord, Economic Science, Monetarism, Rational Expectations, Theorie Neoclassic, Neo-Keynesianism.
\end{abstract}




\title{
LA RESPONSABILIDAD CIVIL AUTOMOVILÍSTICA Y EL ASEGURAMIENTO OBLIGATORIO Y VOLUNTARIO: UN ESTUDIO EN EL DERECHO COLOMBIANO Y ESPAÑOL
}

\author{
Raquel Ceballos Molano
}

\begin{abstract}
RESUMEN
La accidentalidad vial en Colombia es una de las primeras causas de daños corporales y materiales en la población circulante y peatonal, que obliga, al menos en teoría, al propietario o conductor, a responder civilmente de sus consecuencias. Así, este trabajo de tesis plantea el problema de la tipicidad como "actividad peligrosa" y la dispersión normativa derivada de la responsabilidad civil automovilística y los mecanismos de reparación a través del seguro voluntario y obligatorio del automóvil, consagrada en la legislación Civil, Penal, Comercial, Código Nacional de Tránsito, y en Decretos y Resoluciones administrativas, además de la doctrina científica y jurisprudencial, lo que evidencia que el legislativo colombiano no ha prestado atención, desde el punto de vista técnico y jurídico a las políticas de Estado para armonizar en un solo estatuto la responsabilidad derivada de los accidentes de tránsito y su aseguramiento, como si ocurre en el derecho comparado, el ordenamiento jurídico europeo y español.
\end{abstract}

PALABRAS CLAVE: Accidente de transito, responsabilidad civil, seguro obligatorio, soat, seguro automoviles, daño, indemnizacion, reparacion integral.

\section{ABSTRACT}

The road crash in Colombia is one of the first causes of body and materials damages in the circulating and peatonal population, that forces, at least in theory, to the proprietor or conductor, to respond civilly of its consequences. Thus, this thesis work creates the problem of the type like "dangerous activity" and the normative dispersion derived from the automobile civil responsibility and the mechanisms of repair through voluntary and obligatory insurance of the automobile, consecrated in the Civil, Penal, Commercial legislation, National Code of Transit, and in Decrees and administrative Resolutions, in addition to the scientific and jurisprudencial doctrine, which evidence that the legislative Colombian has not paid attention, from the technical and legal point of view to the state policies to harmonize in a single statute the responsibility derived from the traffic accidents and its securing, as if happens in the compared right, the European and Spanish legal ordering.

KEY WORDS: Road accident, civil responsibility, surely obligatory, soat, surely automobiles, damage, indemnification, integral repair. 


\title{
ENVEJECIMIENTO DEL PERSONAL
}

\author{
Sergio Castrillón
}

\section{RESUMEN}

¿De que manera la literatura en ciencias administrativas ha abordado el estudio del envejecimiento del personal? ¿Cuáles son las razones que han motivado este interés en los últimos años? Cuáles son las lógicas subyacentes? Reconociendo las tendencias demográficas mundiales que reflejan el envejecimiento de la población; este artículo procura responder a esta preguntas, a partir de una revisión exhaustiva de las principales publicaciones que se han ocupado del tema en el contexto internacional. Nuestro propósito es aumentar la visibilidad de la problemática y contribuir al debate sugiriendo acciones que revaloricen su dimensión humana.

PALABRAS CLAVE: Administración - Envejecimiento del Personal - Recursos Humanos - Fuerza Laboral - Jubilación - Retiro - Competencias y Desempeño - Motivación - Entrenamiento

\section{ABSTRACT}

How Literature in administrative sciences is boarded the study of the ageing of the personnel? Which are the reasons that have motivated east interest in the last years? Which are the underlying logics? Recognizing world-wide demographic tendencies that reflect the ageing of the population; this article tries to respond to this questions, from an exhaustive revision of the main publications that have taken care of the subject in the international context. Our intention is to increase the visibility of the problematic one and to contribute to the debate being suggested actions that revalue their human dimension.

KEY WORDS: Administration Ageing of the Personnel - Human Resources -Labor Force - Retirement - Competitions and Performance - Motivation - Training 


\title{
RENDICIÓN DE CUENTAS: ESTRATEGIA PARA EL FORTALECIMIENTO DE LA DEMOCRACIA A TRAVÉS DE LA LIMITACIÓN DEL PODER
}

\author{
Carlos Wladimir Gómez Cárdena
}

\begin{abstract}
RESUMEN
Este artículo contiene como apuesta epistemológica la construcción de un modelo genérico de Rendición de Cuentas, el cual puede llegar a ser implementado en las diferentes entidades de naturaleza estatal. A partir de una elaboración teórico - conceptual de la Rendición de Cuentas y de una observación práctica de los primeros ejercicios de rendición de cuentas llevados a cabo en Colombia, se exploran diversas potencialidades e instrumentos que pueden llegar a fortalecer las democracias modernas a través fundamentalmente, de la limitación de los ejercicios de poder. En las democracias modernas son muchos los mecanismos formales de limitación del poder consignados en el conocido sistema de frenos y contrapesos, pero son muy pocos los mecanismos reales. La rendición de cuentas como práctica democrática puede presentarse como uno de estos últimos, en la medida en que la sociedad organizada explore y explote tales potencialidades.
\end{abstract}

PALABRAS CLAVE: Políticas Públicas / Administración Pública / Rendición de Cuentas / Democracia / Limitación del Poder.

\section{ABSTRACT}

This article contains like comely epistemological the construction of a generic model of Accountability, which can arrive to be implemented in the different organizations of state nature. Starting from a theoretical - conceptual elaboration of the Accountability and of a practical observation of the first exercises of Accountability carried out in Colombia, diverse potentialities and instruments are explored that can end up strengthening the modern democracies fundamentally to inclination, of the limitation of the exercises of power. In the modern democracies there are many formal mechanisms of limitation of the power consigned in the well-known system of check and balances, but there are very few real mechanisms. Accountability as democratic practice can be presented like one of these last ones, in the measure in that the organized society explores and exploit such potentialities.

KEY WORDS: Public Policies / Public Administration / Accountability / Democracy / Limitation of the Power. 